\title{
Pressure Dependence of the Chlorine NQR in Chloro Pyridines
}

\author{
K. P. Ramesh, J. Ramakrishna, K. S. Suresh ${ }^{\mathrm{a}}$, and C. Raghavendra Rao ${ }^{\mathrm{a}}$ \\ Department of Physics, Indian Institute of Science, Bangalore 560012, India \\ ${ }^{a}$ Department of Physics, Bangalore University, Bangalore 560056, India \\ Reprint requests to Prof. J. R.; Fax: +91-80-3341683
}

Z. Naturforsch. 55a, 111-116 (2000); received August 25, 1999

Presented at the XVth International Symposium on Nuclear Quadrupole Interactions, Leipzig, Germany, July 25-30, 1999.

The ${ }^{35} \mathrm{Cl} \mathrm{NQR}$ frequency $\left(v_{\mathrm{Q}}\right)$ and spin lattice relaxation time $\left(T_{1}\right)$ in 2,6-dichloropyridine, 2 amino 3,5-dichloropyridine and 6 chloro 2-pyridinol have been measured as a function of pressure up to $5.1 \mathrm{kbar}$ at $300 \mathrm{~K}$, and the data have been analysed to estimate the temperature coefficients of the NQR frequency at constant volume. All the three compounds show a non linear variation of the NQR frequency with pressure which can be described by a $2^{\text {nd }}$ order polynomial in pressure. The rate of change of the NQR frequency with pressure is positive and decreases with increasing pressure. The spin lattice relaxation time $T_{1}$ in all the three compounds shows a small increase with pressure, indicating that the relaxation is mainly due to the torsional motions.

Key words: Chlorine NQR; Spin-Lattice Relaxation Time; Pressure Dependence; 2,6 dichloropyridine; 2-amino 3,5-dichloropyrine; 6-chloro 2-pyridinol.

\section{Introduction}

Our earlier studies on the pressure dependence of the NQR frequency of some solids have shown a nonlinear increase of the NQR frequency with pressure, and also a decrease of the NQR frequency with further increase of pressure in a few cases $[1,2]$. We report here the pressure dependence of the ${ }^{35} \mathrm{Cl} \mathrm{NQR}$ frequency and spin lattice relaxation time $T_{1}$ in 2,6 dichloropyridine, 2 amino 3,5 dichloropyridine and 6 chloro 2 -pyridinol.

\section{Earlier Work}

The NQR frequency of ${ }^{35} \mathrm{Cl}$ in 2,6-dichloropyridine at $77 \mathrm{~K}$ has been reported in [3], and at $300 \mathrm{~K}$ in $[4,5]$. The variation with temperature of the NQR frequency has been studied in [6], and that of $T_{1}$ in [7]. Only a single resonance line was observed in the range 77 to $300 \mathrm{~K}$.

The chlorine NQR of 2 amino 3,5-dichloropyridine and the variation of the NQR frequency and $T_{1}$ with temperature $(77-300 \mathrm{~K})$ were first reported in [8]. Two resonance lines were observed due to the two inequivalent chlorines in the molecule.

The ${ }^{35} \mathrm{Cl} \mathrm{NQR}$ frequency in 6 chloro 2-pyridinol in the range 77 to $300 \mathrm{~K}$ was first reported in [9]. The variation of $T_{1}$ with temperature in the range 77 to $300 \mathrm{~K}$ was studied in [10].

\section{Experimental}

The compounds were obtained from Aldrich Co. USA. The pulsed NQR spectrometer was home made. The pressure was transmitted with liquid paraffin, using a locknut type piston-cylinder device [11] and the teflon cell technique [12]. $T_{1}$ was measured using the inversion recovery sequence $(\pi-\tau-\pi / 2)$. The duration of the free induction decay (FID) was around $150 \mu$ s for all the signals. The NQR frequency and $T_{1}$ were measured with an accuracy of $\pm 0.0005 \mathrm{MHz}$ and $\pm 5 \%$ respectively.

\section{Results and Discussion}

\section{Pressure Dependence of the NQR Frequency}

The effect of lattice vibrations on the average value of the electric field gradient (EFG) [13] can be expressed as

$$
q=q_{0}\left(1-\frac{3}{2}\left\langle\theta^{2}\right\rangle\right),
$$

where $\left\langle\theta^{2}\right\rangle$ is the effective mean square amplitude of the torsional oscillations about the axes of the EFG tensor and $q_{0}$ is the field gradient in the absence of all lattice vibrations. From (1) one obtains

$$
\left(\frac{\partial \ln q}{\partial P}\right)=\left(\frac{\partial \ln q_{0}}{\partial P}\right)_{T}-\frac{3}{2}\left(\frac{\partial\left\langle\theta^{2}\right\rangle}{\partial P}\right)_{T} .
$$




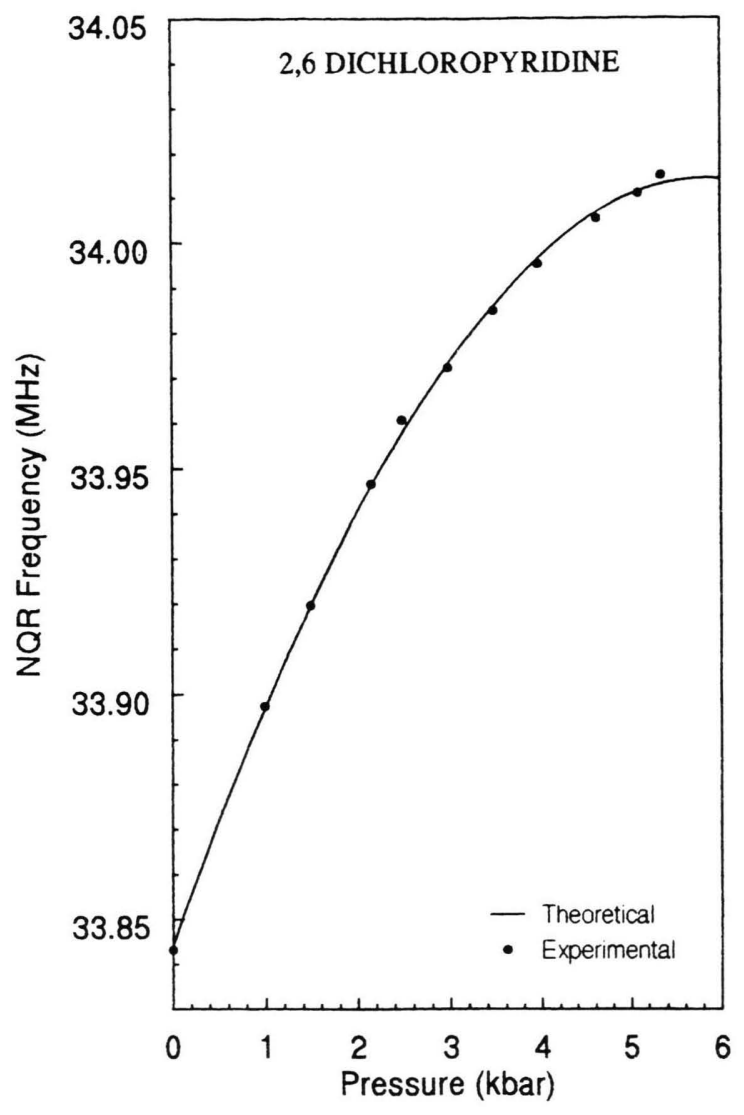

Fig. 1. Pressure dependence of the ${ }^{35} \mathrm{Cl} \mathrm{NQR}$ frequency in 2,6 dichloropyridine.

The first term in (2) is the "static term" and represents the effect of pressure on the static lattice (due to compression) in the absence of all lattice vibrations; the second term, the "dynamic term", represents the effect of pressure on the amplitude of torsional oscillations.

In molecular solids where the EFG is primarily intramolecular in origin, the main contribution to the pressure variation of $\mathrm{NQR}$ frequency comes from changes in the amplitudes of the torsional oscillations. As the amplitudes decrease with pressure, the term $\partial\left\langle\theta^{2}\right\rangle / \partial P$ decreas-

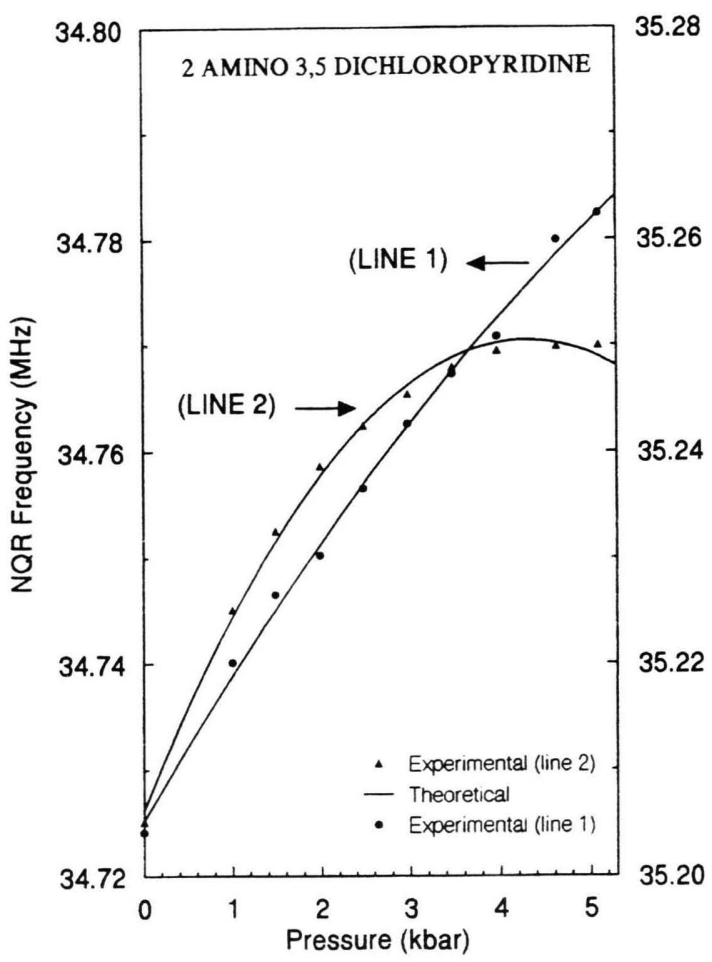

Fig. 2. Pressure dependence of the ${ }^{35} \mathrm{Cl} \mathrm{NQR}$ frequency in 2 amino 3,5 dichloropyridine.

es. Due to the negative sign in (2), the dynamic term results in a positive $(\partial v / \partial P)_{T}$. However, as the pressure is progressively increased, the changes in the torsional amplitudes decrease and the static term becomes more important and may eventually overcome the dynamic contribution.

The pressure dependence of the ${ }^{35} \mathrm{CINQR}$ frequencies at $300 \mathrm{~K}$ in the three compounds is shown in Figures 1-3. The NQR frequencies of the three substances at one bar and $5.1 \mathrm{kbar}$ are given in the Table 1 . The experimental data were fit to a polynomial in pressure:

$$
v(P)=A_{0}+A_{1} P+A_{2} P^{2},
$$

Table 1. The best fit values of Equation (3).

\begin{tabular}{|c|c|c|c|c|c|c|}
\hline \multirow{2}{*}{\multicolumn{2}{|c|}{ Compound }} & \multicolumn{2}{|c|}{ NQR Frequency $(\mathrm{MHz})$} & \multirow{2}{*}{$\begin{array}{l}A_{0} \\
(\mathrm{MHz})\end{array}$} & \multirow{2}{*}{$\begin{array}{l}A_{1} \\
(\mathrm{kHz} \\
\left.\mathrm{bar}^{-1}\right)\end{array}$} & \multirow{2}{*}{$\begin{array}{l}A_{2} \\
\left(\mathrm{kHz}^{2}\right. \\
\left.\mathrm{bar}^{-2}\right)\end{array}$} \\
\hline & & Atm. P & $5.1 \mathrm{kbar}$ & & & \\
\hline \multicolumn{2}{|c|}{ 2,6-dichloropyridine } & 33.8432 & 34.0110 & 33.8449 & 0.05668 & -0.004634 \\
\hline $\begin{array}{l}2 \text { amino } 3,5- \\
\text { dichloropyridine }\end{array}$ & $\begin{array}{l}\text { Line } 1 \\
\text { Line } 2\end{array}$ & $\begin{array}{l}34.7241 \\
35.2041\end{array}$ & $\begin{array}{l}34.7824 \\
35.2499\end{array}$ & $\begin{array}{l}34.7251 \\
35.2051\end{array}$ & $\begin{array}{l}0.01427 \\
0.02002\end{array}$ & $\begin{array}{l}-0.000599 \\
-0.002227\end{array}$ \\
\hline \multicolumn{2}{|c|}{6 chloro 2-pyridinol } & 34.5030 & 34.6818 & 34.5020 & 0.05007 & -0.003014 \\
\hline
\end{tabular}




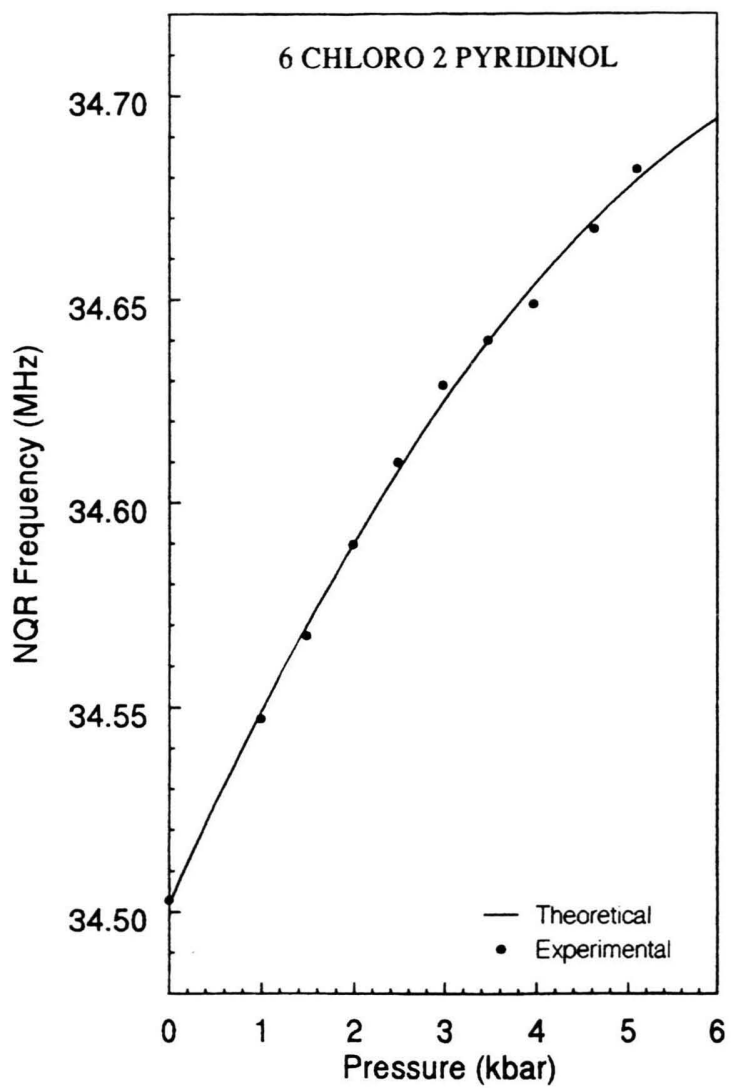

Fig. 3. Pressure dependence of the ${ }^{35} \mathrm{Cl} \mathrm{NQR}$ frequency in 6 chloro 2-pyridinol.

The best fit parameters of (3) are shown in Table 1. Since $A_{2}$ is negative the NQR frequency can decrease if the pressure is increased further. Using the best fit equations, (Figs. 4-6) were obtained $(\partial v / \partial P)_{T}$ is found to be appreciably larger for 2,6-dichloropyridine and 6 chloro 2pyridinol than for 2 amino 3,5-dichloropyridine. The non-linearity of $v(P)$ seems to be due to the opposing nature of the dynamic and static contributions to the pressure dependence and also due to the decrease in the compressibility of solids at higher pressures.

As explained by Bayer [14], the temperature dependence of NQR frequencies arises from the motional averaging of the electric field gradient caused by low-lying librational modes. In the high temperature limit, the temperature dependence of NQR frequencies can be expressed as [15]

$$
\left(\frac{\partial v}{\partial T}\right)_{V}=\sum_{i=x, y}\left(\frac{3 v_{0} k}{4 \pi^{2}}\right) \frac{1}{I_{\mathrm{i}} f_{i}^{2}}
$$

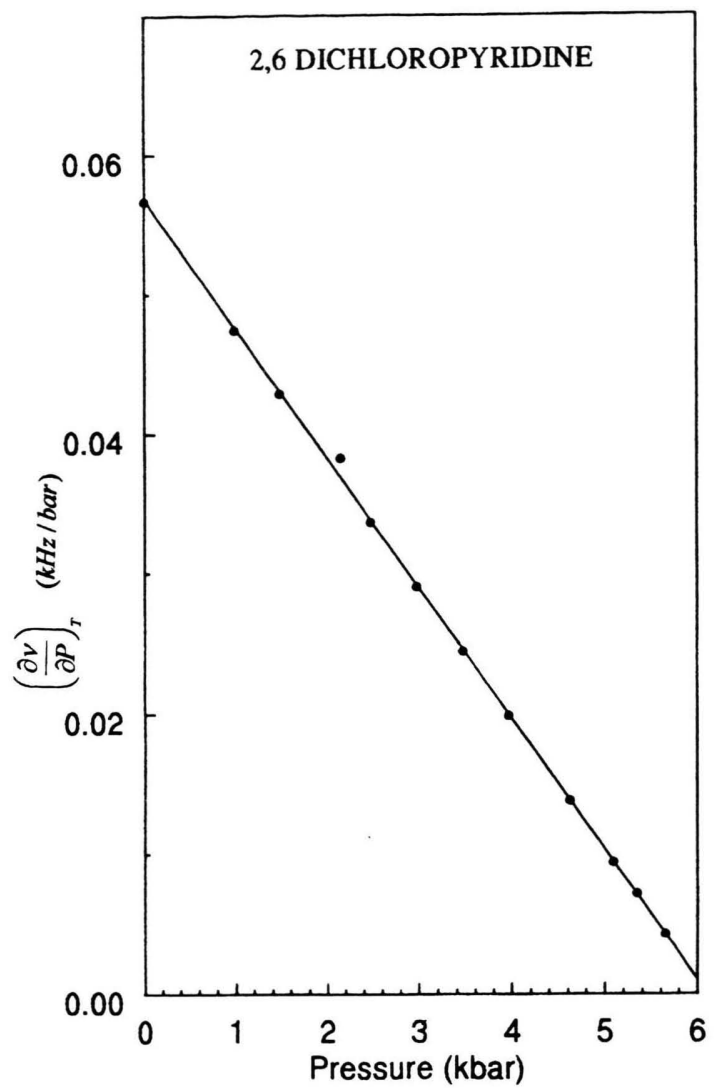

Fig. 4. Variation of $\left(\frac{\partial v}{\partial P}\right)_{T}$ with pressure in 2,6 dichloropy-
ridine.

where $f_{i}$ is the torsional frequency, $I_{\mathrm{i}}$ the moment of inertia of the molecule, $k$ the Boltzmann constant and $v_{0}$ the NQR frequency at $0 \mathrm{~K}$.

Taking into account the effect of volume change due to temperature and pressure, the constant volume temperature derivative of $\mathrm{NQR}$ frequency can also be written as [16]

$$
\left(\frac{\partial v}{\partial T}\right)_{V}=\left(\frac{\partial v}{\partial T}\right)_{P}+\frac{\beta}{\chi}\left(\frac{\partial v}{\partial P}\right)_{T}
$$

where $\beta / \chi$ is the ratio of the macroscopic coefficient of thermal expansion to the isothermal compressibility. In a normal temperature dependence experiment, the temperature coefficient of the resonance frequency at constant pressure is measured. Using (5) it is possible to combine $v(T)$ and $v(P)$ data to estimate the constant volume temperature derivative of the NQR frequency. $(\partial v / \partial T)_{V}$ represents the effect of temperature through molecular motion on the resonance frequency. On the basis of 


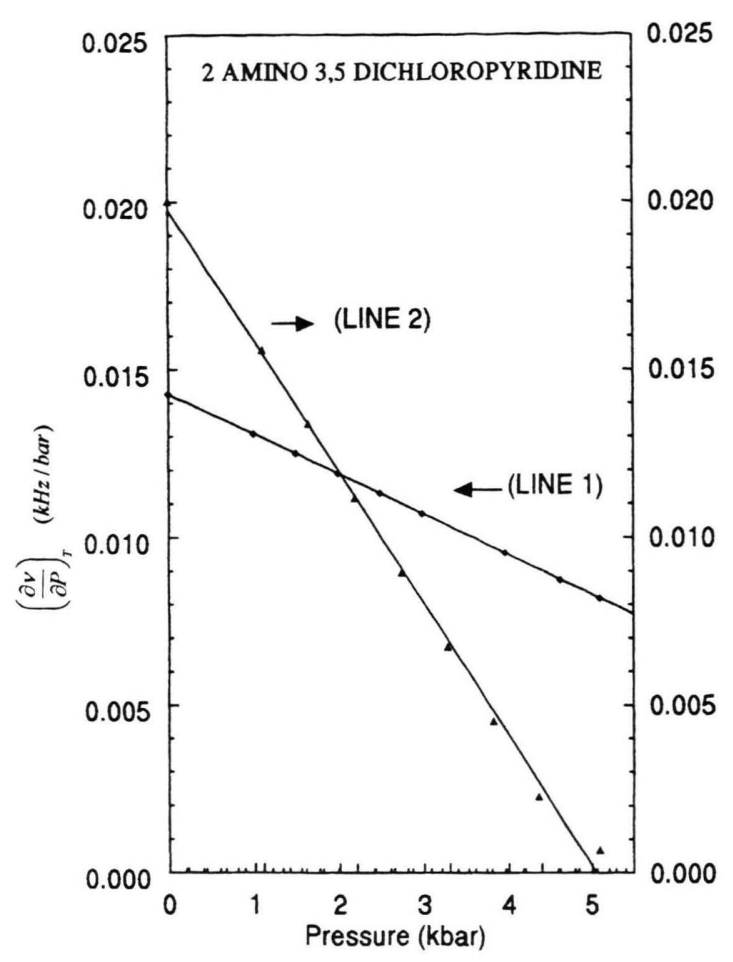
Fig. 5. Variation of $\left(\frac{\partial v}{\partial P}\right)_{T}$ with pressure in 2 amino 3,5 chlo-
ropyridine.

measurements reported for similar substances [16-18], a value of $25 \mathrm{~kg} \mathrm{~cm}^{-2} \mathrm{~K}^{-1}$ has been assumed for the ratio $\beta / \chi$, and using the experimental values of $(\partial v / \partial T)_{P}$ and $(\partial v / \partial P)_{T},(5)$ was solved to estimate the values of $(\partial v / \partial T)_{V}$. These $(\partial v / \partial T)_{V}$ values were used in (4), along with the values of $I_{\mathrm{i}}$ and $v_{0}$ taken from literature $[6,8,9]$ (Table 2), to calculate the torsional frequency for all the compounds, as given in Table 3 . The torsional frequencies are in good agreement with those reported $[6,8,9]$.

\section{Pressure Dependence of the Spin Lattice Relaxation Time $T_{1}$}

Bayer [14] proposed a model that the ${ }^{35} \mathrm{Cl}$ quadrupole spin-lattice relaxation in crystalline $p$-dichlorobenzene and other chlorine substituted aromatic hydrocarbons is governed by random fluctuations in the EFG caused by the torsional oscillations of the molecule. This model was further discussed in detail by Woessner and Gutowsky [19]. Jones et al. [20] have studied the variation of $T_{1}$ with pressure in $p$-dichlorobenzene and 1,3,5 trichlorobenzene where they have found a nonlinear increase in $T_{1}$ with pressure. In the present work, $T_{1}$ in all the three

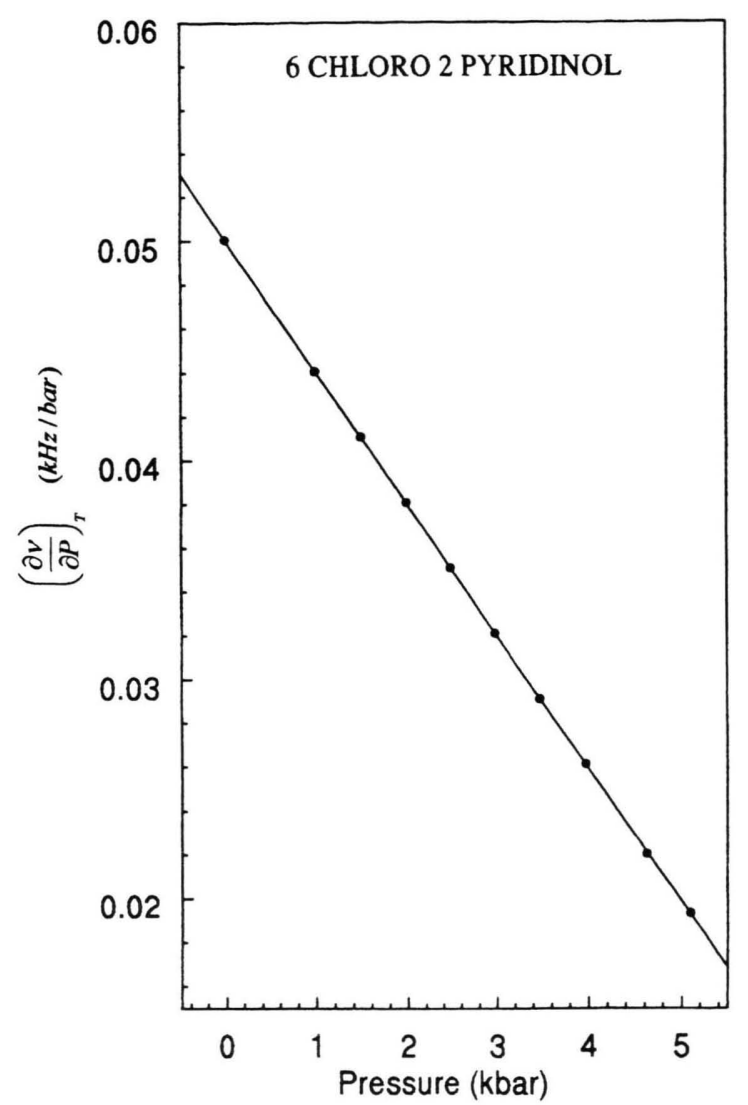

Fig. 6. Variation of $\left(\frac{\partial v}{\partial P}\right)_{T}$ with pressure in 6 chloro 2-pyri-
dinol.

Table 2. Values of $v_{0}$ and $l_{\mathrm{i}}$.

\begin{tabular}{lll}
\hline Compound & $\begin{array}{l}\text { NQR } \\
\text { frequency } \\
\boldsymbol{v}_{0}(\mathrm{MHz})\end{array}$ & $\begin{array}{l}\text { Moment of } \\
\text { inertia } I_{\mathrm{i}} \\
\left(10^{-40} \mathrm{~kg} \mathrm{~cm}^{-2}\right)\end{array}$ \\
\hline 2,6-dichloropyridine & 34.8180 & 1292.5 \\
2 amino 3,5 Line 1 & 35.3323 & 1389.2 \\
dichloropyridine Line 2 & 35.8909 & 1394.5 \\
6 chloro 2-pyridinol & 35.0732 & 918.0 \\
\hline
\end{tabular}

compounds is found to increase slowly with pressure. In case of 2,6-dichloropyridine, $T_{1}$ was found to be $12.3 \mathrm{~ms}$ at atmospheric pressure and $18.1 \mathrm{~ms}$ at $5.65 \mathrm{kbar}$. In 2 amino 3,5-dichloropyridine the values of $T_{1}$ for the two lines are $38.3 \mathrm{~ms}$ and $42.7 \mathrm{~ms}$ at atmospheric pressure and the corresponding values are $53.2 \mathrm{~ms}$ and $57.2 \mathrm{~ms}$ respectively at $5.1 \mathrm{kbar}$. In 6 chloro 2 -pyridinol the value of $T_{1}$ is $9.4 \mathrm{~ms}$ at atmospheric pressure and $16.9 \mathrm{~ms}$ 
Table 3. Temperature and Pressure derivatives of ${ }^{35} \mathrm{Cl} \mathrm{NQR}$.

\begin{tabular}{|c|c|c|c|c|c|}
\hline \multicolumn{2}{|l|}{ Compound } & $\begin{array}{l}(\partial v / \partial T)_{P_{1}} \\
\left(\mathrm{kHz} \mathrm{K}^{-1}\right)\end{array}$ & $\begin{array}{l}(\partial v / \partial P)_{T} \text { at } \\
\mathrm{P}=1 \mathrm{bar}^{-1} \\
\left(\mathrm{~Hz} \mathrm{bar}^{-1}\right)\end{array}$ & $\begin{array}{l}(\partial v / \partial T)_{V} \\
\left(\mathrm{kHz} \mathrm{K}^{-1}\right) \\
\text { Equation }(5)\end{array}$ & $\begin{array}{l}f_{i} \\
\left(\mathrm{~cm}^{-1}\right)\end{array}$ \\
\hline \multicolumn{2}{|c|}{ 2,6-dichloropyridine } & -4.32 & 56.68 & -2.91 & 32.8 \\
\hline $\begin{array}{l}2 \text { amino } 3,5- \\
\text { dichloropyridine }\end{array}$ & $\begin{array}{l}\text { Line } 1 \\
\text { Line } 2\end{array}$ & $\begin{array}{l}-2.93 \\
-2.98\end{array}$ & $\begin{array}{l}14.27 \\
20.02\end{array}$ & $\begin{array}{l}-2.57 \\
-2.48\end{array}$ & $\begin{array}{l}33.9 \\
34.8\end{array}$ \\
\hline \multicolumn{2}{|c|}{6 chloro 2 -pyridinol } & -2.5 & 50.07 & -1.49 & 54.5 \\
\hline
\end{tabular}

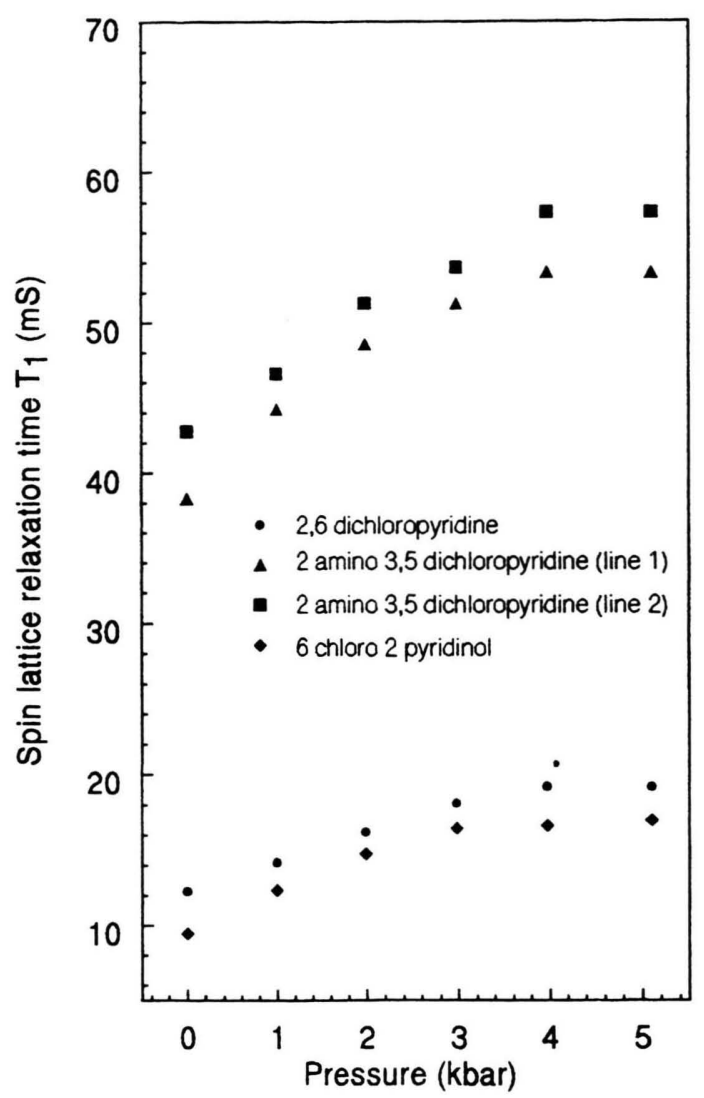

Fig. 7. Pressure dependence of the ${ }^{35} \mathrm{Cl} \mathrm{NQR}$ spin lattice relaxation time in the three pyridines.

at 5.1 kbar. Fig. 7 shows the variation of $T_{1}$ with pressure in the three compounds. Jones et al. [20] have arrived at the following equation, using Bayer's model as developed by Woessner and Gutowsky [19]:

$$
\frac{\mathrm{d} \ln T_{1}}{\mathrm{~d} P}=-\frac{\zeta}{\beta T}\left(m+\frac{\mathrm{d} \ln T_{1}}{\mathrm{~d} \ln T}\right),
$$

where $\zeta$ is the volume compressibility given as $\zeta=$ $-\mathrm{d}(\ln V) / \mathrm{d} P$ and $\beta$ is the cubic coefficient of thermal ex-
Table 4. Comparison of pressure and temperature derivatives of $T_{1}$.

\begin{tabular}{llll}
\hline Compound & $\frac{\mathrm{d} \ln T_{1}}{n}$ & $\frac{\mathrm{d} \ln T_{1}}{\mathrm{~d} P}$ & $m$ \\
& $\left(\mathrm{kbar}^{-1}\right)$ & $\mathrm{d} \ln T$ & \\
\hline 2,6-dichloropyridine & 0.13 & -2.38 & 2.30 \\
2 amino 3,5 Line 1 & 0.23 & -2.31 & 2.29 \\
dichloropyridine Line 2 & 0.17 & -2.18 & 2.18 \\
6 chloro 2-pyridinol & 0.34 & -2.16 & 1.84 \\
\hline
\end{tabular}

pansion given as $\beta=\mathrm{d}(\ln V) / \mathrm{d} T$. In molecular crystals in which relaxation is governed by random fluctuations in the EFG due to the torsional modes, $\mathrm{d}\left(\ln T_{1}\right) / \mathrm{d} P$ is positive and $d\left(\ln T_{1}\right) / d \ln T$ is negative. The values of $\mathrm{d}\left(\ln T_{1}\right) / \mathrm{d} \ln T$ typically lie in the range -2 to -3 [21]. When the spin lattice relaxation is due to torsional motions, the variation of $T_{1}$ with temperature follows the equation [19]

$$
T_{1}^{-1}=a T^{m} \text {. }
$$

The variation of $T_{1}$ with temperature has been studied in [7] for 2,6-dichloropyridine in [8] for 2 amino 3,5-dichloropyridine, and in [10] for 6 chloro 2-pyridinol. Table 4 gives the pressure and temperature derivatives of $T_{1}$ for the three compounds along with the $\mathrm{m}$ values. The values of $m$ are found to lie within 1.8 and 2.4. The signs of these differentials are consistent with (6), and $\mathrm{d} \ln T_{1}$ / $\mathrm{d} P$ is as expected for the case where $T_{1}$ is mainly due to torsional oscillations. Thus the torsional motions are responsible for the relaxation, resulting in the weak pressure dependence of $T_{1}$.

\section{Conclusion}

The NQR frequency in the pyridines studied increases nonlinearly with pressure, which is indicative of an increased contribution from the "static" distortion at higher pressures. Constant volume Bayer terms are calculat- 
ed, which show good agreement between theoretical and experimental values. The experimental $T_{1}$ shows a small increase with pressure, which implies that torsional oscillations are mainly responsible for the relaxation.

[1] T. V. Krishna Moorthy, V. Krishnan, and J. Ramakrishna, Molecular Physics 55, 121 (1985).

[2] V. Krishnan, K. R. Sridharan, and J. Ramakrishna, Polyhedron 4, 739 (1985).

[3] M. Redshaw, J. Chem. Soc. Perkin Trans. II, 110 (1976).

[4] P. Bucci, Cecchi, and A. Colligiani, J. Amer. Chem. Soc. 87, 3027 (1965).

[5] E. A. C. Lucken and C. Mazeline, Proc. Coll. Ampere 13, 235 (1965).

[6] S. P. Basavaraju, N. Devaraj, A. Indumathy, K. R. Sridharan, and J. Ramakrishna, J. Solid State Chem. 47, 236 (1983)

[7] J. Srinivas, Ph.D. Thesis, (1995). I.I.Sc., Bangalore 12.

[8] Mohammed Hossein Ala Amjadi, K. P. Ramesh, N. Rajendra Kumar, and J. Ramakrishna, Magnetic Resonance in Chemistry 37, 693 (1999).

[9] S. P. Basavaraju, N. Devaraj, A. Indumathy, K. R. Sridharan, and J. Ramakrishna, Aust. J. Chem. 35, 211 (1982).

[10] M. Hossein, Ph.D. Thesis (1997). I.I.Sc., Bangalore 12.

[11] K. V. Ramanathan and R. Srinivasan, J. Phys. E11, 480 (1978)

\section{Acknowledgement}

This work was supported by a grant from Council of Scientific and Industrial Research, New Delhi. - 110001.

[12] A. Jayaraman, A. R. Hutson, J. H. McFee, A. S. Coriell, and R. G. Maines, Rev. Scient. Instrum. 38, 44 (1967)

[13] G. C. Gillies and R. J. C. Brown, Can J. Chem. 54, 2266 (1976).

[14] H. Bayer, Z. Phys. 130, 227 (1951).

[15] D. F. Cooke and R. L. Armstrong, Can. J. Phys. 49, 238 (1971).

[16] T. Kushida, G. B. Benedek, and N. Bloembergen, Phys. Rev. 104, 1364 (1956).

[17] P. W. Bridgman, Proc. Amer. Acad. Arts. Sci. 76, 55 (1948).

[18] Landolt-Bornstein, Zahlenwerte und Funktionen, Springer-Verlag, Berlin, 1951, p. 2221.

[19] D. E. Woessner and Gutowsky, J. Chem. Phys. 39, 440 (1963).

[20] L. V. Jones, M. Sabir, and J. A. S. Smith, J. Chem. Soc. Faraday II 74, 1723 (1978).

[21] I. Kjunzel, V. A. Mokeeva, G. B. Soifer, and I. G. Shaposhnikov, J. Magn. Res. 20, 394 (1975). 